\title{
Quantum HF/DFT-Embedding Algorithms for Electronic Structure Calculations: Scaling up to Complex Molecular Systems
}

\author{
Max Rossmannek \\ IBM Quantum, IBM Research - Zurich, 8803 Rüschlikon, Switzerland \\ Panagiotis Kl. Barkoutsos \\ IBM Quantum, IBM Research - Zurich, 8803 Rüschlikon, Switzerland \\ Pauline J. Ollitrault \\ IBM Quantum, IBM Research - Zurich, 8803 Rüschlikon, \\ Switzerland; Laboratory of Physical Chemistry, ETH Zurich, 8093 Zürich, Switzerland \\ Ivano Tavernelli* \\ IBM Quantum, IBM Research - Zurich, 8803 Rüschlikon, Switzerland
}

(Dated: September 7, 2020)

\begin{abstract}
In the near future, material and drug design may be aided by quantum computer assisted simulations. These have the potential to target chemical systems intractable by the most powerful classical computers. However, the resources offered by contemporary quantum computers are still limited, restricting the chemical simulations to very simple molecules. In order to rapidly scale up to more interesting molecular systems, we propose the embedding of the quantum electronic structure calculation into a classically computed environment obtained at the Hartree-Fock (HF) or Density Functional Theory (DFT) level of theory. We achieve this by constructing an effective Hamiltonian that incorporates a mean field potential describing the action of the inactive electrons on a selected Active Space (AS). The ground state of the AS Hamiltonian is determined by means of the Variational Quantum Eigensolver (VQE) algorithm. With the proposed iterative DFT embedding scheme we are able to obtain energy correction terms for a single pyridine molecule that outperform the Complete Active Space Self-Consistent Field (CASSCF) results regardless of the chosen AS.
\end{abstract}

\section{INTRODUCTION}

Quantum chemistry simulations allow the prediction of important chemical processes throughout, for instance, the elucidation of reaction mechanisms by means of the calculation of ground or excited state electronic structure properties [1]. A variety of research and industrial such as chemical catalysis, material design, drug discovery and photo-chemical applications for solar energy conversion, just to name a few 2, 3, could take advantage of these methods. Since the development of the first computers, the research on quantum chemistry has blossomed and a large variety of algorithms has been developed aspiring to achieve more accurate solutions of Schrödinger's equation. However, despite many theoretical and algorithmic advances the solutions of many interesting and relevant problems in chemistry and physics remain out of reach due to the inherent exponential scaling of the Hilbert space associated with the electronic structure calculations. While several approximate methods have been developed in the past to circumvent this issue, these often break down when considering strongly correlated systems such as transition metal complexes [4 and complicated catalytic processes 5 . In the past decades, quantum computing has emerged as a new potential computational

* ita@zurich.ibm.com paradigm for the solution of many problems in chemistry and physics for which classical algorithms have an unfavorable scaling. In particular, quantum computing has been shown to be a useful resource in a variety of research areas such as chemistry [4, 6], drug discovery [7, strongly correlated systems [8, 9, field theory [10, 11, material science [12] and many others.

Despite these recent advances and the possibility to execute calculations on quantum devices (e.g., Ref. [13]), the application of quantum algorithms is still in its infancy. In fact, most of the research in chemistry relies on hybrid quantum-classical algorithms [14, which use highly optimized classical (number crunching) functionalities together with quantum algorithms for the representation and optimization of the system wavefunction. The most well known quantum chemistry algorithm that provides the means to leverage state-of-the-art quantum hardware is the Variational Quantum Eigensolver (VQE) [15.

For the representation of the many-body wavefunction in quantum circuits, some of the approaches derived in quantum chemistry can be mapped directly to quantum computing. In particular, the Hartree-Fock (HF) method has proven to pose a useful starting point for the mapping of electronic structure problems in the qubit space using the so-called second quantization formalism. Among the most commonly used post-HF expansions of the many-electron wavefunction in quantum computing is the Coupled Cluster (CC) Ansatz [16 18, which allows 
for a systematic and controlled inclusion of higher order configurations starting from the uncorrelated HF Slater determinant. Several quantum implementation of CC have already been introduced in the literature [15, 1925], including schemes for the optimization of the oneelectron molecular basis functions [9, 26]. In addition to the classically-inspired expansions, pure native quantum representations of the many-electron wavefunctions that can be better optimized for the available quantum hardware platforms have been proposed [21, 27. The combination of the VQE algorithm with the different wavefunction Ansätze showed already interesting results in the calculation of ground [19, 27 30 ] and excited state properties [15, 31 40] of simple molecules (up to a few atoms). However, this protocol does not allow to scale to larger systems using the currently available classical simulators of quantum circuits (limited to a maximum of about 50 qubits) or the available quantum computers (also limited to a few tens of qubits). Therefore, in order to leverage the potential advantage of the available quantum algorithm we explore the possibility of an embedding scheme in which only a portion of the full system is represented by the high-level quantum computing approach, while the rest is treated with an efficient but (necessarily) approximated classical representation of the electronic structure, such as HF or Density Functional Theory (DFT). This embedding approach is of particular relevance when the complex, highly correlated, subsystem can be localized in a well defined subspace of the complete set of one-electron orbitals used to represent the many-electron wavefunction. In this case, an accurate description of the electronic structure is obtained at lower cost, namely $\mathcal{O}\left(N_{q c}^{4}\right)$ for the quantum computing (qc) subsystem and $\mathcal{O}\left(N_{\text {env }}^{2}\right)$ to $\mathcal{O}\left(N_{e n v}^{3}\right)$ for the environment (env) (with $N_{e n v}=N_{t o t}-N_{q c}$ ), instead of the $\mathcal{O}\left(N_{\text {tot }}^{4}\right)$ scaling when no embedding is used.

In this work, we propose HF and DFT-based quantum embedding schemes based on the well known notion of an Active Space (AS) 41, 42, which defines the set of active orbitals described by the quantum algorithm. To this end, we will construct an effective Hamiltonian which incorporates a mean field potential of the inactive electrons and, thus, fully replaces the explicit mapping of the corresponding orbitals in the quantum register. The quantum algorithm is therefore restricted to a subset of active orbitals, which, however, feel the presence of the environment through the action of the mean field potential generated by the inactive electrons of the environment. Similar approaches of the HF embedding have been proposed in the literature [43, 44 mainly based on Dynamical Mean Field Theory (DMFT) 45] and Density Matrix Embedding Theory (DMET) [46 for the highlevel description of the subsystem. The latter aims at a similar HF embedding scheme. However, while the focus of its authors was the development of a self-consistent HF embedding approach [47, in this work we will only consider iterative embedding within the framework of DFT. Concerning the DMFT approach, this is based on Green's function techniques and therefore it is not particularly suited for the kind of molecular applications of interest to this work. Additionally, during the preparation of this paper another related approach appeared in the literature [48. In this case, the authors propose a DFT embedding scheme similar to ours, which however uses a different Ansatz to resolve the double counting problem of the correlation terms. Furthermore, they do not update the embedding potential in a self-consistent manner as we do in our work.

This paper is organized as follows. In Section III we outline the theory and the implementation of the proposed AS schemes for quantum electronic structure calculations embedded in HF and DFT. We split the derivation into two parts: one for the HF embedding scheme, and one for the DFT embedding scheme. Section III lists the technical details of our numerical methods. In Section IV we present and discuss results for both embedding schemes applied to a few molecular test systems, namely $\mathrm{H}_{2} \mathrm{O}, \mathrm{N}_{2}, \mathrm{O}_{2}, \mathrm{CH}_{2}$ and pyridine. Section $\mathrm{V}$ summarizes and concludes.

\section{THEORY}

In this work, we propose two embedding schemes for quantum electronic structure algorithms based on $\mathrm{HF}$ and Kohn-Sham (KS) DFT Molecular Orbitals (MOs). The subsystem solved by means of the quantum approach (such as quantum Unitary Coupled Cluster Singles and Doubles (q-UCCSD) 1, 15, 21]) is embedded in the potential generated by the environment (i.e., the remaining electrons), which is computed within the $\mathrm{HF}$ or DFT framework. Our solutions are based on the Range-Separation (RS) technique for the two-electron integrals 49, which allows for a rigorous partitioning of the problem into a subsystem (i.e., the AS) and its environment. If this partitioning is done wisely, we can achieve a good level of accuracy for many properties of interest while significantly reducing the costs of the calculation. Furthermore, in the case of the DFT embedding scheme (which is the main target of this work) we will extend the algorithm to include the self-consistent optimization of the embedding potential, leading to more accurate energies and densities. In the following we will call active electrons the electrons that are part of the AS, while the remaining ones will be referred to as inactive.

\section{A. Hartree-Fock Embedding}

In this first section, we derive the so-called inactive Fock operator. The goal of this operator is to embed the quantum computation into a classically computed environment treated at the HF level of theory, through the notion of an AS. While this method is not new and different variants of it have been implemented before in other software packages [48, 50, 51, in the following we sum- 


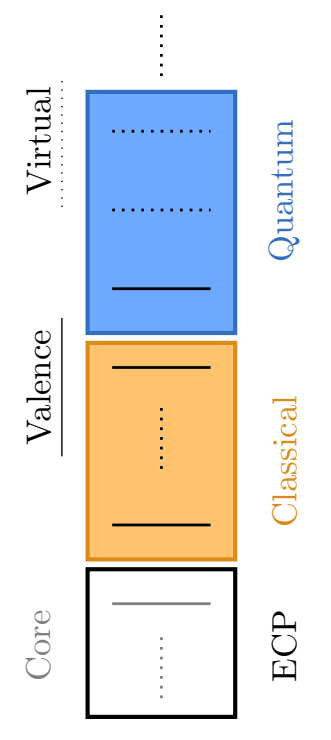

Figure 1. Separation of the MOs into active and inactive components. The active orbitals (blue box) are mapped onto the qubit space and treated with the q-UCCSD approach while the inactive ones (orange box) are part of the HF/DFT embedding and are evaluated classically. Effective Core Potentials (ECPs) can be used in replacement of all inactive core electrons (white box) with the aim of reducing the computational cost.

marize the key concepts that are needed for its implementation within the framework of quantum computing in QISKIT [52]. This section also lays down the fundamentals for the implementation of the DFT embedding scheme presented in Section IIB.

The benefit of this embedding scheme lies in outsourcing the calculation of the inactive electrons to the classical HF driver while the quantum computation is restricted to the AS. In this way, less qubit resources are necessary to investigate the electronic energy of a molecular system, making the entire calculation much more efficient while keeping a good level of accuracy. Fig. 1 depicts the separation of the orbitals into the active and inactive spaces.

The total electronic energy, $E$, is defined by expectation value of the system Hamiltonian, $\hat{\mathcal{H}}$,

$$
E=\langle\Psi|\hat{\mathcal{H}}| \Psi\rangle=\sum_{p q} h_{p q} D_{p q}+\frac{1}{2} \sum_{p q r s} g_{p q r s} d_{p q r s},
$$

where $\Psi$ is the wavefunction, $h_{p q}$ and $g_{p q r s}$ are the oneand two-electron integrals, respectively, and $D$ and $d$ are the one- and two-particle density matrices.

To achieve the implementation of the HF embedding we split the one-electron density into an active and inactive part, $D=D^{A}+D^{I}$. In the MO basis, the latter simplifies to $D_{i q}^{I}=2 \delta_{i q}$, where we use Helgaker's notation of indices [42] in which $i, j, k, l$ denote inactive, $u, v, x, y$ denote active and $p, q, r, s$ denote general MOs. As shown in the Appendix A 1 inserting this into Eq. (1) leads to

$$
E=E^{I}+\sum_{u v} F_{u v}^{I} D_{u v}^{A}+\frac{1}{2} \sum_{u v x y} g_{u v x y} d_{u v x y}^{A}
$$

where we define the inactive Fock operator,

$$
F_{p q}^{I}=h_{p q}+\sum_{i}\left(2 g_{i i p q}-g_{i q p i}\right),
$$

and the inactive energy,

$$
E^{I}=\sum_{j} h_{j j}+F_{j j}^{I}=\frac{1}{2} \sum_{i j}\left(h_{i j}+F_{i j}^{I}\right) D_{i j}^{I} .
$$

Comparing Eq. (1) and Eq. (2) we observe the following differences. In Eq. (2) the inactive Fock operator, $F^{I}$, replaces the one-electron integrals, $h_{p q}$, the active oneand two-electron density matrices, $D^{A}$ and $d^{A}$, replace $D$ and $d$, and the constant energy offset, $E^{I}$, is added.

Therefore, the Hamiltonian which we will simulate on the quantum computer $(q c)$ takes the form

$$
\hat{\mathcal{H}}_{q c}=\sum_{u v} F_{u v}^{I} \hat{a}_{u}^{\dagger} \hat{a}_{v}+\sum_{u v x y} g_{u v x y} \hat{a}_{u}^{\dagger} \hat{a}_{v}^{\dagger} \hat{a}_{x} \hat{a}_{y},
$$

where $\hat{a}_{u}^{\dagger}$ and $\hat{a}_{u}$ are the creation and annihilation Fermionic operators (later mapped to the qubit space using the parity transformation [53]). Note that all indices are restricted to the AS, significantly reducing the required quantum resources.

The extension for the unrestricted formalism is obtained in a similar manner and is outlined in Appendix A 2.

\section{B. Density Functional Theory Embedding}

In order to extend the embedding to work with DFT we need to introduce a RS of the two-electron integrals, $g_{p q r s}$ [49. To this extent, we split the two-electron operator, $\hat{g}_{p q}$, into a Long-Range (LR) and a Short-Range (SR) part,

$$
\hat{g}_{p q}=\frac{1}{\left|\hat{r}_{p}-\hat{r}_{q}\right|}=\hat{g}_{p q}^{\mu, \mathrm{LR}}+\hat{g}_{p q}^{\mu, \mathrm{SR}},
$$

where $\mu$ is the RS parameter of unit $a . u .^{-1}$. This is necessary in order to avoid a double counting of the correlation terms which are present in both, DFT and Wave Function Theory (WFT). Since DFT is known to be accurate for SR interactions [49, we can use it to treat the SR part while the LR interactions are calculated with WFT.

Our derivation of the following equations follows that of Hedegård et al. [54] closely. Additionally, we provide our detailed derivations in Appendix B.

With the RS of the two-electron integrals in place, we can split the total electronic energy into two terms,

$$
E=E_{\mathrm{WFT}}^{\mu, \mathrm{LR}}+E_{\mathrm{coul}+\mathrm{xc}, \mathrm{DFT}}^{\mu, \mathrm{SR}} .
$$


Note that Eq. 77 provides an adiabatic connection between the pure DFT and the pure WFT solutions through the coupling parameter, $\mu$. However, in order to simplify the notation we drop the superscript $\mu$ since it is anyways implied by the separation into LR and SR.

Analogous to Section IIA, we can introduce an AS in the WFT part,

$$
E=E_{\mathrm{WFT}}^{I, \mathrm{LR}}+E_{\mathrm{WFT}}^{A, \mathrm{LR}}+E_{\mathrm{coul}+\mathrm{xc}, \mathrm{DFT}}^{\mathrm{SR}} .
$$

Note that the difference between Eq. (2) and Eq. (8) is that WFT only treats the LR part. Thus, the inactive Fock operator, defined in Eq. (3), becomes

$$
F_{p q}^{I, \mathrm{LR}}=h_{p q}+\sum_{i}\left(2 g_{i i p q}^{\mathrm{LR}}-g_{i q p i}^{\mathrm{LR}}\right) .
$$

In order to properly combine the SR-DFT and LRWFT calculations we need to handle the non-linearity of $E_{\mathrm{coul}+\mathrm{xc}, \mathrm{DFT}}^{\mathrm{SR}}=E_{\mathrm{coul}+\mathrm{xc}}^{\mathrm{SR}}$ on the electronic density, $\rho$,

$$
E_{\mathrm{coul}+\mathrm{xc}}^{\mathrm{SR}}[\rho+\Delta \rho] \neq E_{\mathrm{coul}+\mathrm{xc}}^{\mathrm{SR}}[\rho]+E_{\mathrm{coul}+\mathrm{xc}}^{\mathrm{SR}}[\Delta \rho],
$$

where $\Delta \rho$ is the correction to the density obtained from the WFT calculation. However, a linear model can be obtained with the following approximation,

$$
\begin{aligned}
& E_{\mathrm{coul}+\mathrm{xc}}^{\mathrm{SR}}[\rho+\Delta \rho]-E_{\mathrm{coul}+\mathrm{xc}}^{\mathrm{SR}}[\rho] \\
& \approx \int \frac{\delta E_{\mathrm{coul}+\mathrm{xc}}^{\mathrm{SR}}}{\delta \rho(\vec{r})}[\rho] \Delta \rho(\vec{r}) \mathrm{d} \vec{r} .
\end{aligned}
$$

The right hand side of Eq. 111) can then be expressed in terms of the Coulomb integrals,

$$
j_{p q}^{\mathrm{SR}}=\left\langle\phi_{p}\left|\frac{\delta E_{\mathrm{coul}}^{\mathrm{SR}}}{\delta \rho(\vec{r})}[\rho]\right| \phi_{q}\right\rangle=\sum_{r s} g_{p q r s}^{\mathrm{SR}} D_{r s}
$$

and the exchange integrals,

$$
\nu_{\mathrm{xc}, p q}^{\mathrm{SR}}=\left\langle\phi_{p}\left|\frac{\delta E_{\mathrm{xc}}^{\mathrm{SR}}}{\delta \rho(\vec{r})}[\rho]\right| \phi_{q}\right\rangle=\nu_{\mathrm{xc}, p q}^{\mathrm{SR}}[\rho],
$$

as,

$$
\int \frac{\delta E_{\mathrm{coul}+\mathrm{xc}}^{\mathrm{SR}}}{\delta \rho(\vec{r})}[\rho] \Delta \rho(\vec{r}) \mathrm{d} \vec{r}=\sum_{p q}\left(j_{p q}^{\mathrm{SR}}+\nu_{\mathrm{xc}, p q}^{\mathrm{SR}}\right) \Delta D_{p q} .
$$

Because of the non-linearity of Eq. (12b) the density needs to be updated in an iterative, self-consistent manner. Therefore, we define the density and the density matrix at the iteration step $i$ as,

$$
\begin{gathered}
\rho^{(i+1)}=\rho^{(i)}+\Delta \rho^{(i)}, \\
D_{p q}^{(i+1)}=D_{p q}^{(i)}+\Delta D_{p q}^{(i)} .
\end{gathered}
$$

This leads to the final form of the total electronic energy,

$$
\begin{aligned}
E & =\frac{1}{2} \sum_{i j}\left(h_{i j}+F_{i j}^{I, \mathrm{LR}}\right) D_{i j}^{I} \\
& +E_{\mathrm{xc}}^{\mathrm{SR}}\left[\rho^{(i)}\right]+\frac{1}{2} \sum_{i j} j_{i j}^{I, \mathrm{SR}} D_{i j}^{I} \\
& -\sum_{u v}\left[\left(\frac{1}{2} j_{u v}^{A,(i), \mathrm{SR}}+\nu_{\mathrm{xc}, u v}^{\mathrm{SR}}\left[\rho^{(i)}\right]\right) D_{u v}^{A,(i)}\right. \\
& \left.+\left(F_{u v}^{I, \mathrm{LR}}+j_{u v}^{I, \mathrm{SR}}+j_{u v}^{A,(i), \mathrm{SR}}+\nu_{\mathrm{xc}, u v}^{\mathrm{SR}}\left[\rho^{(i)}\right]\right) D_{u v}^{A,(i+1)}\right] \\
& +\frac{1}{2} \sum_{u v x y} g_{u v x y}^{\mathrm{LR}} d_{u v x y}^{A,(i+1)},
\end{aligned}
$$

where we have ordered the terms such that the top line contains all contributions which remain constant for the duration of the whole iterative procedure, the second and third lines correspond to the SR-DFT, and the remaining lines correspond to the LR-WFT energy terms, respectively.

Fig. 2 summarizes the implementation of this DFT embedding scheme. The initialization step includes all the pre-calculations and the computation of the constant inactive LR energy contribution (first line of Eq. (15)). The resulting energy terms of steps (1) and (2) in Fig. 2 correspond to lines two and three, and four and five of Eq. 15, respectively. These two calculations iterate, upon exchanging the active electronic density, $\rho^{A}$, until the total electronic energy reaches convergence.

\section{NUMERICAL METHODS}

The HF and DFT embedding schemes have been implemented in the latest development version 0.8 of QISKIT Aqua Chemistry. The source code is made available in the Github repository [55. For the classical computing backend we choose PYSCF [56] since it allows quick prototyping within Python, the same programming language used for QISKIT.

All the results presented hereafter are obtained by means of diagonalizing the Hamiltonian with the ExactEigensolver [57] algorithm as implemented in QISKIT. In the case of the non-iterative HF embedding scheme, we also run VQE simulations with the statevector backend [55]. This backend implements an exact, i.e. noiseless, simulation of the quantum circuit and, thus, is expected to converge to the same result as the ExactEigensolver approach when a suitable wavefunction Ansatz is chosen. In addition, for some selected systems (see below) we also perform noisy VQE calculations, referred to as QASM simulations, using noise models corresponding to the two IBM Q devices ibmq_almaden and ibmq_boeblingen. 


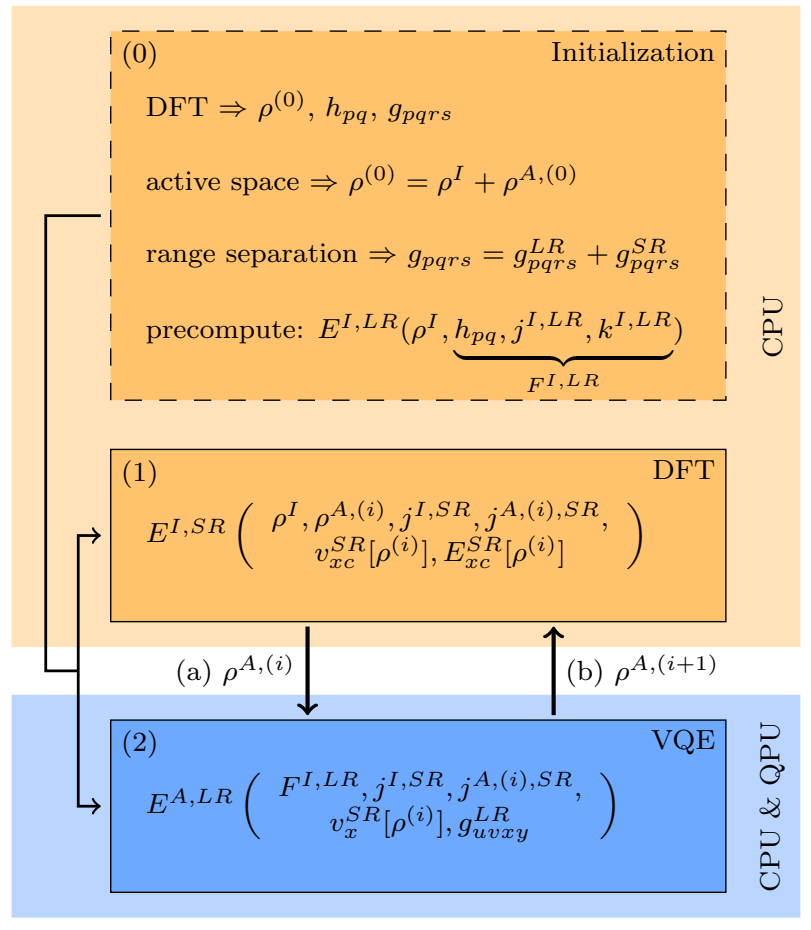

Figure 2. Illustration of the DFT embedding scheme. During initialization (0), a DFT calculation of the full system is performed using a classical code, providing the initial density, $\rho^{(0)}$, and the one- and two- electron integrals, $h_{p q}$ and $g_{p q r s}$. The density is then split into inactive, $I$, and active parts, $A$, and the two-electron integrals are separated into long-range, $L R$, and short-range, $S R$, components. In step (1) the inactive SR energy contribution is calculated at the DFT level of theory. The resulting 'active' density component $(a)$ is used in step (2) to initialize the VQE optimization. This returns the active LR energy contribution and the updated electronic density, which is used as a new input for the DFT calculation, (b). Steps (1) and (2) are repeated until convergence.

\section{A. Hartree-Fock Embedding}

In all simulations using the HF embedding, we use the parity fermions-to-qubits mapping [53] and the qUCCSD Ansatz 21] for the representation of the electronic wavefunction. Furthermore, qubits are tapered off [58 in order to maximally reduce the computational costs. The classical optimizers L-BFGS-B [59] and SLSQP [60] are used for the optimization of the VQE parameters in the case of noiseless and noisy simulations, respectively.

Ten qubits are needed for the simulation of the selected systems with the noisy VQE algorithm, after application of the parity transformation. These could be reduced to six after tapering off [58]. Thus, only a subset of the total qubits available on ibmq_almaden and ibmq_boeblingen was used to perform the simulations. These subsets of qubits are $1,2,3,8,7,6$ on ibmq_almaden and $3,2,1,6,7,8$ on ibmq_boeblingen. The connection between the first and last qubits of the sequence allows for a more efficient implementation of the quantum algorithm after compilation [52]. This avoids the extensive use of SWAP gates to achieve coupling between qubits, which are not directly connected in the chip.

\section{B. Density Functional Theory Embedding}

In all DFT embedding applications we use the RS-XCF (Range-Separated Exchange-Correlation Functional) ldaerf scheme 61, 62 as implemented in the xcfun library 63] for the separation of the LDA (Local Density Approximation) functional into its short and long range components (see Section II B). This approach achieves the splitting of the two-electron integrals by means of the error function which is a common approach in RS-DFT [49, 64, 65. The use of the LDA functional is solely motivated by the current technical limitations of the PySCF code. Future extensions to allow the use of arbitrary DFT functionals are under investigation. Nonetheless, the proposed scheme is fully independent from the nature of the selected functional and all applications presented in the following should be considered as proof-of-principle demonstrations extendable to any type of DFT functional.

In-line with the previous simulations with the HF embedding scheme, we make use of the $6-31 \mathrm{G}^{*}$ basis set, the parity mapping [53, and the q-UCCSD Ansatz [21] for the representation of the electronic wavefunction.

\section{RESULTS AND DISCUSSION}

We present the results obtained with the proposed HF and DFT embedding schemes (Section II A and II B, respectively). Using the HF embedding approch, we investigate a variety of small molecules highlighting the broad applicability of the procedure. On the other hand, the better accuracy of DFT calculations over HF will enable us to scale up to larger molecular systems such as the heterocyclic pyridine molecule.

\section{A. Hartree-Fock Embedding}

We test the HF embedding scheme on several molecular systems, including $\mathrm{H}_{2} \mathrm{O}, \mathrm{N}_{2}, \mathrm{CH}_{2}$ and $\mathrm{O}_{2}$. To simplify the discussion of the results, we take the water molecule as a benchmark system, while all other systems will be presented in the full paper.

In the case of $\mathrm{H}_{2} \mathrm{O}$, we investigate the effect of the basis set on the accuracy of the ground state energy by increasing its size from STO-3G [66 to $6-31 \mathrm{G}^{*} 67$. and cc-pVTZ [68. Furthermore, we consider several ASs ranging from the minimum of two electrons in two MOs $(\mathrm{CAS}(2,2))$ all the way up to ten electrons in ten MOs $(\operatorname{CAS}(10,10))$. 


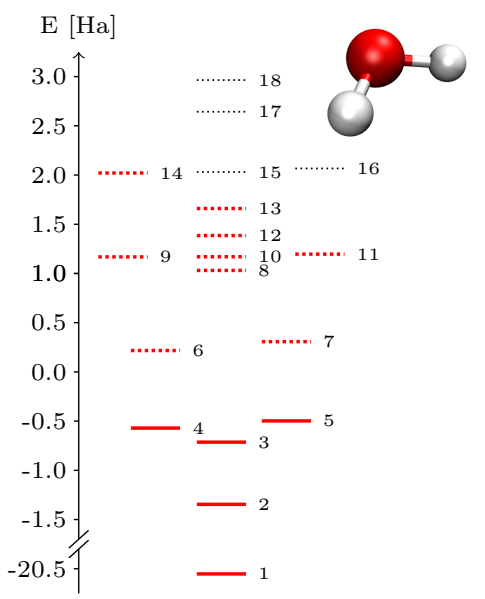

Figure 3. Energy diagram of the HF-MOs of $\mathrm{H}_{2} \mathrm{O}$ in the 6$31 \mathrm{G}^{*}$ basis. Solid lines correspond to occupied MOs while dotted lines represent virtual ones. The MOs corresponding to the red lines (1 to 14 ) are visualized in Fig. 4.

\section{A benchmark system: water}

As a first test case, we apply the HF embedding scheme to the case of a single water molecule using the $6-31 \mathrm{G}^{*}$ basis. Fig. 3 presents an energy diagram of the HF-MO energies and the active HF-MOs are shown in Fig. 4.

In Fig. 5 we summarize the main results. In general, the q-UCCSD Ansatz applied in this work reproduces ground state energies in qualitative good agreement with the classical CASCI approach [56] (orange crosses in Fig. 5). This shows that the inclusion of merely the single and double excitations is sufficient to obtain the Full Configuration Interaction (FCI) accuracy within a given AS for a simple system such as $\mathrm{H}_{2} \mathrm{O}$. Furthermore, most statevector-based VQE calculations (brown bars in Fig. 5) converge to the exact solutions (blue bars in Fig. 5). The CASSCF [56] results (green circles in Fig. 5) are consistently lower (or equal) in energy than the CASCI approach since this method also optimizes the orbital coefficients, which are kept fixed in the CASCI method.

As expected [70, 71, we also observe that the energy corrections obtained with the embedding method do not trivially depend on the chosen AS, in particular with respect to the number of occupied versus unoccupied orbitals (see the trends in Fig. 5). Thus, it is not possible to draw a general conclusion about the optimal selection of the AS since this is highly dependent on the molecule, the basis set, and the underlying optimization method. In practice, other choices than the selection of the MOs around the Fermi level could be also considered. Indeed, tools have been developed which aim at automatizing the selection of the optimal AS [29, 71-74]. However, the combination of these approaches with the proposed embedding scheme goes beyond the scope of this work and will become the subject of future investigations.

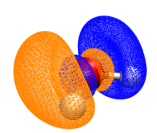

12
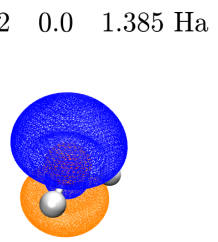

$9 \quad 0.0 \quad 1.169 \mathrm{Ha}$
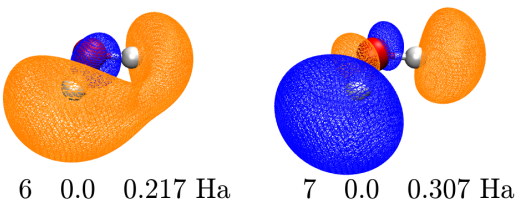

$\begin{array}{lll}6 & 0.0 & 0.217 \mathrm{Ha}\end{array}$

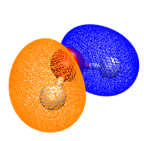

$\begin{array}{lll}3 & 2.0 & -0.714 \mathrm{Ha}\end{array}$

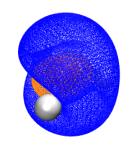

13

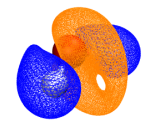

$10 \quad 0.0 \quad 1.170 \mathrm{Ha}$

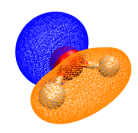

$4 \quad 2.0 \quad-0.571 \mathrm{Ha}$
$11 \quad 0.0 \quad 1.196 \mathrm{Ha}$

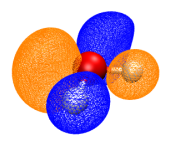

$8 \quad 0.0 \quad 1.031 \mathrm{Ha}$

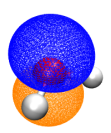

$\begin{array}{lll}5 & 2.0 & -0.498 \mathrm{Ha}\end{array}$

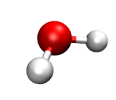

$1 \quad 2.0-20.552 \mathrm{Ha}$

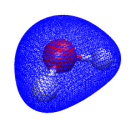

$2 \quad 2.0 \quad-1.346 \mathrm{Ha}$
Figure 4. Visualization of the active HF-MOs of water in the $6-31 \mathrm{G}^{*}$ basis. The orbital coefficients, energies and occupation numbers are obtained with PYSCF using a RHF calculation. All orbitals are rendered using VMD 69] at an isovalue of 0.075 . The three numbers below each orbital correspond to the HF-MOs index (as indicated in Fig. 3), the occupation number, and the orbital energy. The first MO is essentially identical to the oxygen's 1s orbital and completely hidden by the red sphere representing the oxygen atom.

In order to assess the influence of the quantum sampling error on the accuracy of the embedding scheme, we perform some additional calculations using the QASMbased VQE with a realistic description of the hardware noise. The results of these simulations for the $\operatorname{CAS}(2$, 5) system are also included in Fig. 5 (black plus and triangles). The main hardware noise sources are related to infidelity of the qubit operations, qubit decoherence, readout errors, and statistical sampling of the expectation values. Concerning this last point, in our simulations we will use the standard value of 8192 measurements for 


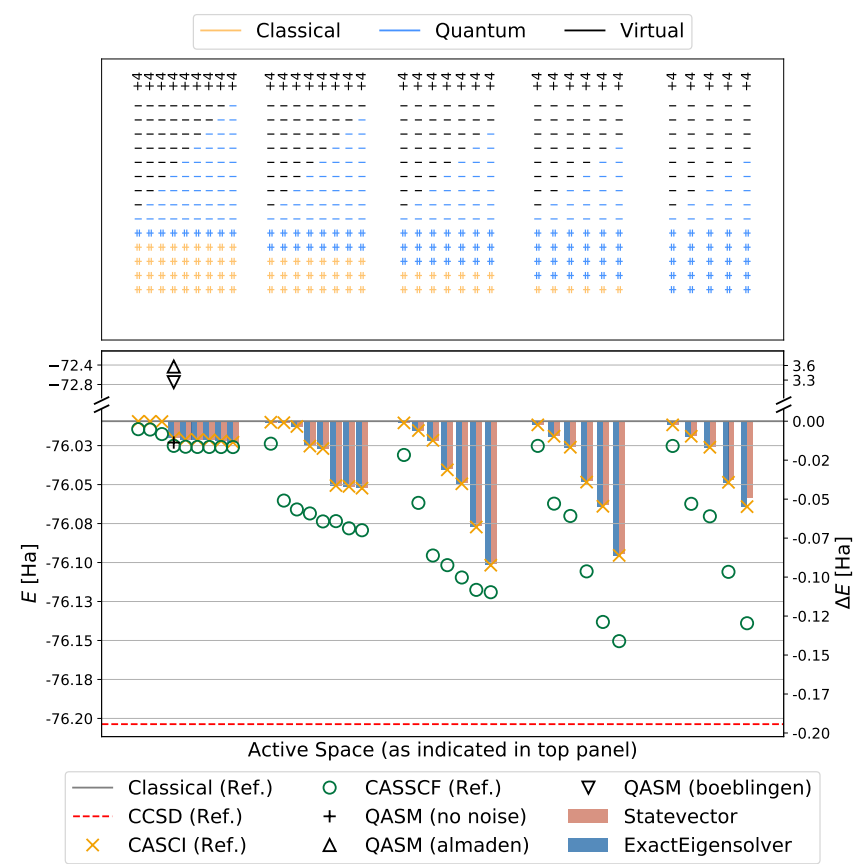

Figure 5. Electronic structure energies (in Hartree) of a $\mathbf{H}_{2} \mathbf{O}$ molecule obtained using the HF embedding scheme for different choices of the AS. The classical HF reference was obtained with RHF in the $6-31 \mathrm{G}^{*}$ basis using PYSCF. All energy differences relative to this value are shown on the $y$-axis on the right (lower panel). The CCSD, CASCI and CASSCF references were also computed with PYSCF. The corresponding ASs are depicted in the upper panel. The +4 above the ASs indicates that 4 additional virtual orbitals are omitted from the visualization. The coloring follows the same scheme used in Figs. 1 and 2 orange for the inactive DFT orbitals that defines the embedding, blue the orbitals belonging to the $\mathrm{AS}$, and in black the remaining virtual ones.

each expectation value. First, we start with a test QASMbased VQE simulation in which we artificially repress all noise sources. For the selected AS, this 'noiseless' simulation converges to the qualitatively correct energy value (black plus in Fig. 5), in agreement with the corresponding statevector-based VQE calculation. Upon addition of hardware noise models of the ibmq_almaden and ibmq_boeblingen devices the quality of the results drops dramatically, as indicated by the black triangles in Fig. 5 . Despite the careful selection of qubits on each device (cf. Section III A, the circuit depth required for the implementation of the q-UCCSD-based embedding calculation is still beyond the limits of what can be executed (and simulated) on state-of-the-art quantum computers. This is reflected by the very large deviations between the VQE energy and the actual ground state energy. (In fact, the results are less accurate than the initial HF energies.)

In general, these results on 'noisy' simulations confirm the inadequacy of the q-UCCSD Ansatz for experiments on hardware, as reported for instance in Ref. [75, and alternative wave function Ansätze need to be investigated.
To this extent, a particle conserving heuristic Ansatz can become a viable option aiming at significantly shorter circuit depths [21, 27, 76].

In the Supplementary Information (SI) we also provide the outcomes obtained with the STO-3G and cc-pVTZ basis sets. In the case of STO-3G the largest AS includes all available orbitals resulting in the recovery of the exact CCSD energy $(\operatorname{CAS}(10,7)$ in Fig. S1). Furthermore, we also perform another set of QASM-based VQE simulations for the $\operatorname{CAS}(8,5)$ case (black plus and triangles in Fig. S1) which do not differ qualitatively from the results discussed previously for $\operatorname{CAS}(2,5)$ in the $6-31 \mathrm{G}^{*}$ basis. In the case of the cc-pVTZ basis set, the quantitative energy correction terms are smaller than for $6-31 \mathrm{G}^{*}$ due to the much larger total number of molecular orbitals (while keeping a constant AS size).

\section{Towards more complex systems}

In addition to the $\mathrm{H}_{2} \mathrm{O}$ benchmark system, we apply the $\mathrm{HF}$ embedding scheme to a series of other small molecules: $\mathrm{N}_{2}, \mathrm{CH}_{2}$ and $\mathrm{O}_{2}$, using the $6-31 \mathrm{G}^{*}$ basis set. These systems vary in size, spin multiplicity of the ground state and elemental composition and serve as a demonstration of the wide applicability of our approach. We summarize the main results of these calculations in Table II where we also introduce the following measure for the energy correction

$$
\varepsilon_{A S}^{e m b}=\frac{E_{A S}^{e m b}-E_{\mathrm{HF}}}{E_{\mathrm{CCSD}}-E_{\mathrm{HF}}}[\%],
$$

where the subscript, $A S$, and superscript, emb, indicate the method used to treat the AS and the 'embedding' method, respectively. Thus, $E_{\mathrm{q}-\mathrm{UCCSD}}^{\mathrm{HF}}$ denotes the $\mathrm{HF}$ embedding energies corresponding to Eq. (2) and $E_{\mathrm{CASSCF}}^{\mathrm{HF}}$ denotes the classical reference energies obtained by CASSCF. When the superscript is omitted, this implies that no 'embedding' method is used, resulting in $E_{\mathrm{CCSD}}\left(E_{\mathrm{HF}}\right)$ i.e., the CCSD (HF) energy with all MOs as part of the AS. Thus, Eq. 16 defines a system-independent quality measure allowing us to quantify the quality of the HF embedding. We choose the CCSD energy as our 'exact' reference because it defines a lower bound on the energy achievable with the q-UCCSD Ansatz when all orbitals (and not just a subset) are included in the AS.

As evident from inspection of Table I, the HF embedding leads to significant energy corrections for all of the investigated systems, leading to the recovery of $18-48 \%$ of the energy difference between CCSD and HF (ignoring the case of water in the STO-3G basis where all MOs are active). As discussed previously, the nature of the AS that gives rise to the largest energy correction cannot be predicted by simple inspection of the MOs involved. 
Table I. Summary of the HF-based embedding calculations yielding the largest energy correction, $\varepsilon_{A S}^{e m b}$ (cf. Eq. 16 ). For systems with a non-singlet ground state we denote the number of active $\alpha$-electrons explicitly as $\operatorname{CAS}\left(N_{\mathrm{el}}\left(N_{\mathrm{el}}^{\alpha}\right), \overline{\left.N_{\mathrm{mo}}\right)}\right.$. All reference energies were obtained with the same initial HF densities used for the embedding calculation and were computed with PYSCF. Energy values are in Ha.

\begin{tabular}{lllrrrrr}
\hline \multirow{2}{*}{ System } & Basis Set & Active Space & $E_{\mathrm{q}-\mathrm{UCCSD}}^{\mathrm{HF}}\left(\varepsilon_{\mathrm{q}-\mathrm{UCCSD}}^{\mathrm{HF}}\right)$ & $E_{\mathrm{CASCI}}^{\mathrm{HF}}$ & $E_{\mathrm{CASSCF}}^{\mathrm{HF}}\left(\varepsilon_{\mathrm{CASSCF}}^{\mathrm{HF}}\right)$ & $E_{\mathrm{HF}}$ & $E_{\mathrm{CCSD}}$ \\
\hline $\mathrm{H}_{2} \mathrm{O}$ & $\mathrm{STO}-3 \mathrm{G}$ & $\mathrm{CAS}(10,7)$ & $-75.009(100.0)$ & -75.009 & $-75.009(100.0)$ & -74.961 & -75.009 \\
$\mathrm{H}_{2} \mathrm{O}$ & $6-31 \mathrm{G}^{*}$ & $\mathrm{CAS}(6,10)$ & $-76.102(47.7)$ & -76.102 & $-76.119(56.4)$ & -76.009 & -76.204 \\
$\mathrm{H}_{2} \mathrm{O}$ & $\mathrm{cc}-\mathrm{pVTZ}$ & $\mathrm{CAS}(6,10)$ & $-76.108(17.9)$ & -76.108 & $-76.191(47.5)$ & -76.058 & -76.338 \\
$\mathrm{~N}_{2}$ & $6-31 \mathrm{G}^{*}$ & $\mathrm{CAS}(8,10)$ & $-109.033(29.2)$ & -109.033 & $-109.114(55.5)$ & -108.943 & -109.251 \\
$\mathrm{CH}_{2}$ & $6-31 G^{*}$ & $\mathrm{CAS}(6(4), 10)$ & $-38.959(37.6)$ & -38.959 & $-38.992(70.3)$ & -38.921 & -39.022 \\
$\mathrm{O}_{2}$ & $6-31 G^{*}$ & $\mathrm{CAS}(8(5), 10)$ & $-149.707(27.8)$ & -149.707 & $-149.749(40.7)$ & -149.616 & -149.943 \\
\hline
\end{tabular}

\section{B. Density Functional Theory Embedding}

KS-DFT is the classical effective single-particle method of choice when dealing with molecular systems since both, energies and geometries, are systematically improved over-HF. In order to fully appreciate the advantages of the DFT embedding scheme, we need to scale up the size of the system beyond what we have done so far with the HF approach. To this end, after a short test on a single water molecule, we introduce a more challenging validation test focusing on the pyridine molecule.

\section{The water molecule}

As expected, due to the relatively small size of this system, we do not expect important improvements using DFT over the results obtained with HF reported in Section IV A. The same also applies to the shape of the $\mathrm{KS}-\mathrm{MOs}$ and the corresponding energy diagram, which do not differ significantly from the HF ones shown in Fig. 3 and 4 . The results obtained with the DFT embedding are summarized in Fig. 6 for different choices of the AS. To select the optimal value of the RS parameter, $\mu$ (cf. Eq. (6)), we performed a scan ranging from 0.01 to 500 finding an optimal value at $\mu_{\text {opt }}=7.25$. The number of iterations to reach convergence of the DFT embedding (see Fig. 2) varies for the different values of $\mu$. In average, 4 iterations were sufficient to meet the energy threshold of $10^{-10} \mathrm{Ha}$. We then investigate the behavior of the energy correction in the neighborhood of $\mu_{\text {opt }}$ for different sizes of the AS (i.e., number of MOs) and number of active electrons. The energy curves as a function of the RS parameter, $\mu$ (cf. Eq. (6)), are grouped into panels with constant numbers of active electrons $(2$, 4, 6 and 8, respectively). In each panel, the results are shown for different number of active MOs as indicated in the legend.

As expected, for values of $\mu$ tending to zero, we recover the plain LDA-DFT result (the dotted, grey line in Fig. 6). Furthermore, in the limit of large $\mu$, the energy converges towards the value obtained with the $\mathrm{HF}$ embedding scheme discussed in the previous section. For convenience, the energy values evaluated in this limit $(\mu \rightarrow \infty)$, as well as the corresponding CASSCF energies, are reported at $\mu=1000$ in Fig. 6. These data points are indicated with different marker symbols, yet color-coordinated to match the corresponding ASs.

In order to allow a quantitative comparison of the DFT embedding results with the ones obtained with the HF embedding and the classical reference, CASSCF, we define a new system-independent measure, similar to Eq. (16), which reads

$$
\tilde{\varepsilon}_{A S}^{e m b}=\frac{\tilde{E}_{A S}^{e m b}-\tilde{E}_{\mathrm{DFT}}}{E_{\mathrm{CCSD}}-\tilde{E}_{\mathrm{DFT}}}[\%],
$$

where the $\sim$ indicates energies computed with $\mathrm{KS}-\mathrm{MOs}$ (instead of HF-MOs) and the DFT reference energy, $\tilde{E}_{\mathrm{DFT}}$, is the value obtained using the LDA/VWN functional (corresponding to the embedding energy in the limit $\mu \rightarrow 0)$. Following the same notation of Eq. 16 , the subscript, $A S$, and superscript, emb, indicate the method used to treat the AS and the 'embedding' method, respectively. This leads to the DFT embedding energies, $\tilde{E}_{\mathrm{q}-\mathrm{UCCSD}}^{\mathrm{DFT}}$ (cf. Eq. 15 ), the HF embedding energies based on KS-MOs, $\tilde{E}_{\mathrm{q}-\mathrm{UCCSD}}^{\mathrm{HF}}$ (corresponding to the embedding energy in the limit $\mu \rightarrow \infty$, cf. Eq. (2)), and the classical reference based on CASSCF, $\tilde{E}_{\mathrm{CASSCF}}^{\mathrm{HF}}$. With the help of this measure, we observe (see Table II) a significant improvement of the DFT embedding energy correction over the HF one by about $13 \%$ compared to the common reference CCSD value. However, the success of the method is highly dependent on the value of the RS parameter, $\mu$. Thus, we recommend a sweep over a reasonable range of $\mu$ values when investigating new systems and/or properties thereof as done in this work.

\section{The pyridine molecule}

The pyridine molecule $\left(\mathrm{C}_{5} \mathrm{H}_{5} \mathrm{~N}\right)$ provides a more challenging test case for the validation of the RS-DFT embedding scheme. Fig. 7 presents an energy diagram of the KS-MO energies of pyridine in the $6-31 \mathrm{G}^{*}$ basis and Fig. 8 shows the relevant set of orbitals which make up our investigated ASs. 

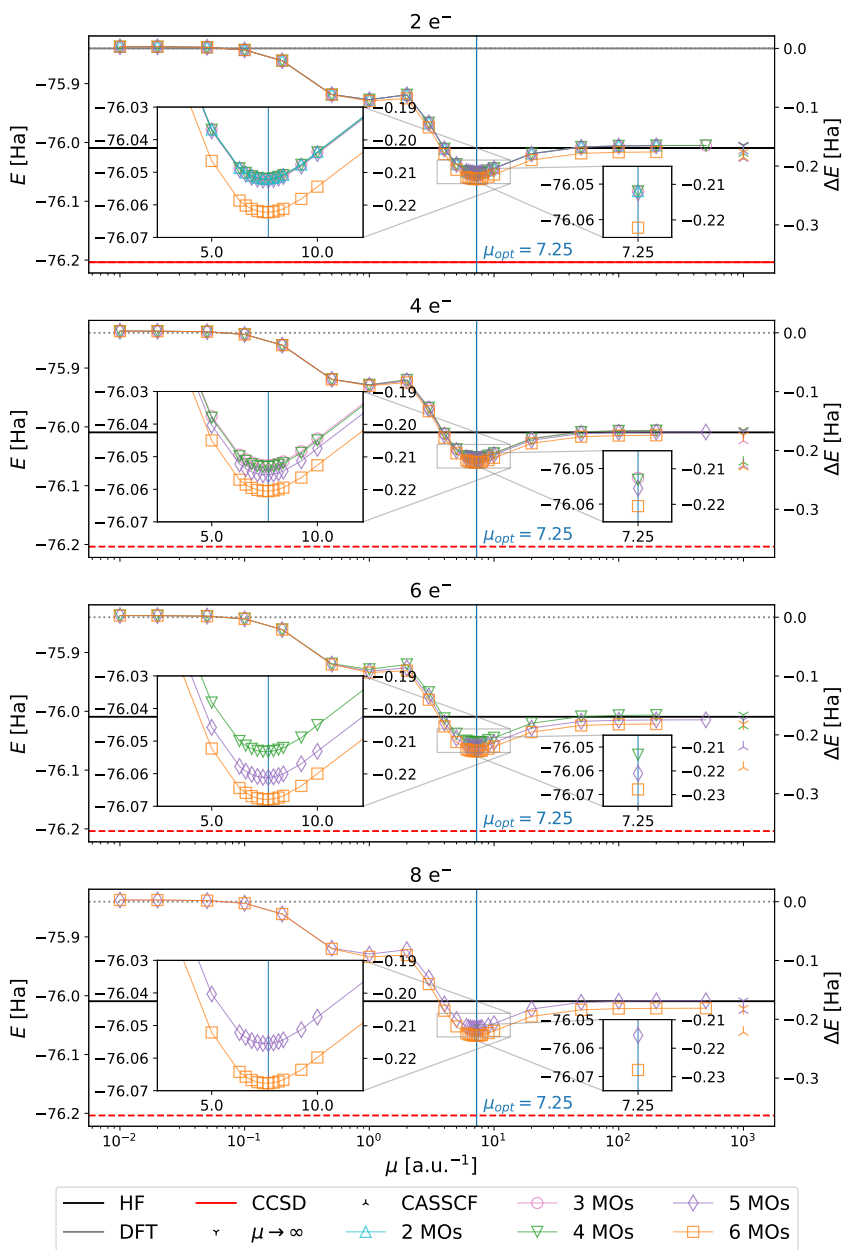

Figure 6. Electronic structure energies (in Hartree) of a $\mathbf{H}_{2} \mathbf{O}$ molecule obtained using the DFT embedding scheme for a different number of active electrons. Each panel groups results obtained for different numbers of active electrons (from the top: $2,4,6,8)$ while the number of MOs in the AS is color-coded (see legend). The classical references were computed using PySCF and the 6-31G* basis. The initial density was obtained using RKS-DFT with the LDA/VWN XCF (grey, dotted). For completeness, we also provide the HF and CCSD references in solid black and dashed red, respectively. The range separation parameter, $\mu$, is varied along the $x$ axis. Reference values for $\mu \rightarrow \infty$ and for the CASSCF results are included at $\mu=10^{3}$ with different marker symbols and colorcoded according to the respective ASs.

In Fig. 9 we summarize the main results obtained for pyridine with the DFT embedding scheme. The organization of the four panels follows the same logic used for the case of the water molecule reported in Fig. 6 the colors refer to the different sizes of the AS (i.e., number of MOs in the AS) while each panel deals with a different number of active electrons. The insets in each panel show a zoom of the region around the optimal value, $\mu_{\text {opt }}=5.25$. This differs from the one optimized for water, pointing towards a system dependence of $\mu_{\text {opt }}$, which, however, remains constant across different ASs for the same sys-

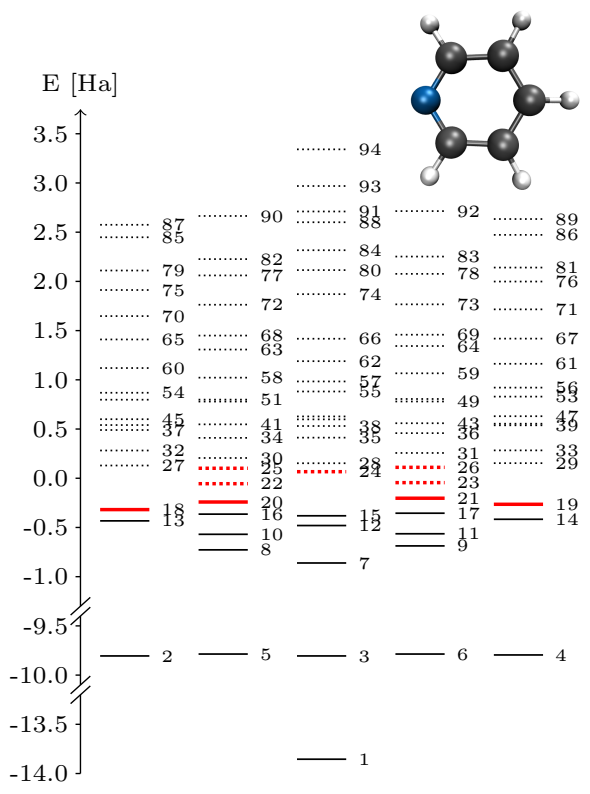

Figure 7. Energy diagram of the KS-MOs of pyridine in the 6-31G* basis. Solid lines correspond to occupied MOs while dotted lines represent virtual ones. The MOs corresponding to the red lines (18 to 26 ) are visualized in Fig. 8 To improve readability, every second MO label between 40 and 54 is omitted.

tem. A similar effect using the RS-LDA functional was already reported in Ref. 65. However, the extension to more accurate XCFs can alleviate this dependence to some extent 65]. As for the case of the water molecule, in average 4 iterations between the classical driver (dealing with the DFT environment) and the quantum processor (dealing with the AS) were needed to achieve convergence of the system energy within a threshold of $10^{-10} \mathrm{Ha}$, independently from the value of $\mu$.

Considering the accuracy of the final energies, we observe that the DFT embedding results outperform the corresponding HF ones (at $\mu=1000$ ) by about $250 \mathrm{mHa}$, reaching $\tilde{\varepsilon}_{\mathrm{q}-\mathrm{UCCSD}}^{\mathrm{DF}}$ values (cf. Eq. (17)) around $60 \%$ regardless of the number of active electrons. However, we have to stress the importance of a parametric sweep of $\mu$ again, since the success of the DFT embedding strongly depends on the chosen value. Additionally, a smarter choice of the AS where the MO are not restricted around the Fermi level may further improve the obtained energy corrections.

An encouraging observation that we can draw from inspection of Fig. 9 is that for a system of the size of pyridine (with 42 electrons) we can already outperform the classical CASSCF approach for all AS choices. In fact, we obtain energy corrections of around $60 \%$ compared to values around $48 \%$. This is a very promising result, which highlights the benefits that can be obtained from the combination of RS embedding approaches with quantum electronic structure algorithms. We summarize these results in Table II] where we compare the energy 
Table II. Summary of the DFT-based embedding calculations yielding the largest energy correction, $\tilde{\varepsilon}_{A S}^{m b}$ (cf. Eq. 17]). See also Fig. 6 and 9 Reference energies were obtained with the same initial DFT densities used for the embedding calculation and were computed with PYSCF. All systems were modeled with the 6-31G* basis. Energy values are in Ha.

\begin{tabular}{llrrrrrr}
\hline System & \multirow{2}{*}{ Active Space } & $\mu_{\mathrm{opt}}$ & $\tilde{E}_{\mathrm{q}-\mathrm{UCCSD}}^{\mathrm{DFT}}\left(\tilde{\varepsilon}_{\mathrm{q}-\mathrm{UCCSD}}^{\mathrm{DFT}}\right)$ & $\tilde{E}_{\mathrm{q}-\mathrm{UCCSD}}^{\mathrm{HF}}\left(\tilde{\varepsilon}_{\mathrm{q}-\mathrm{UCCSD}}^{\mathrm{HF}}\right)$ & $\tilde{E}_{\mathrm{CASSCF}}^{\mathrm{HF}}\left(\tilde{\varepsilon}_{\mathrm{CASSCF}}^{\mathrm{HF}}\right)$ & $\tilde{E}_{\mathrm{DFT}}$ & $E_{\mathrm{CCSD}}$ \\
\hline $\mathrm{H}_{2} \mathrm{O}$ & $\mathrm{CAS}(2,6)$ & 7.25 & $-76.062(61.0)$ & $-76.016(48.4)$ & $-76.026(51.1)$ & -75.840 & -76.204 \\
$\mathrm{H}_{2} \mathrm{O}$ & $\mathrm{CAS}(4,6)$ & 7.25 & $-76.061(60.7)$ & $-76.014(47.8)$ & $-76.069(62.9)$ & -75.840 & -76.204 \\
$\mathrm{H}_{2} \mathrm{O}$ & $\mathrm{CAS}(6,6)$ & 7.25 & $-76.068(62.6)$ & $-76.021(49.7)$ & $-76.095(70.1)$ & -75.840 & -76.204 \\
$\mathrm{H}_{2} \mathrm{O}$ & $\mathrm{CAS}(8,6)$ & 7.25 & $-76.068(62.6)$ & $-76.021(49.7)$ & $-76.062(61.0)$ & -75.840 & -76.204 \\
pyridine & $\mathrm{CAS}(2,6)$ & 5.25 & $-246.902(58.3)$ & $-246.647(41.0)$ & $-246.717(45.8)$ & -246.042 & -247.517 \\
pyridine & $\mathrm{CAS}(4,6)$ & 5.25 & $-246.918(59.4)$ & $-246.663(42.1)$ & $-246.747(47.8)$ & -246.042 & -247.517 \\
pyridine & $\mathrm{CAS}(6,6)$ & 5.25 & $-246.943(61.1)$ & $-246.689(43.9)$ & $-246.761(48.7)$ & -246.042 & -247.517 \\
pyridine & $\mathrm{CAS}(8,6)$ & 5.25 & $-246.943(61.1)$ & $-246.688(43.8)$ & $-246.745(47.7)$ & -246.042 & -247.517 \\
\hline
\end{tabular}
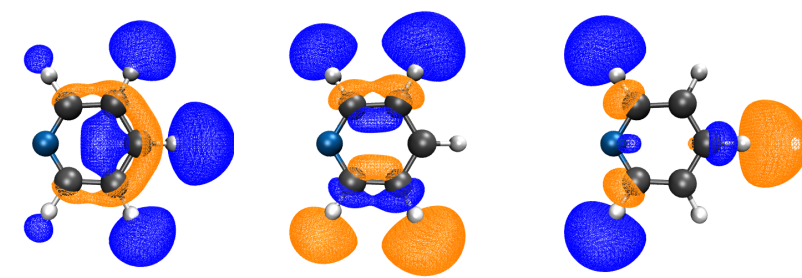

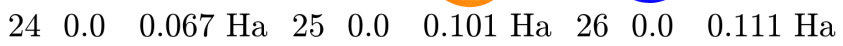
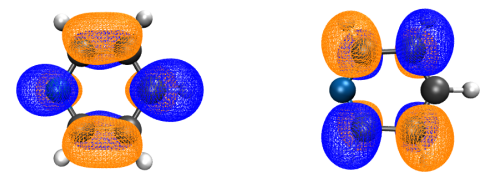

22

$\begin{array}{lllll}0.0 & -0.055 \mathrm{Ha} & 23 & 0.0 & -0.045 \mathrm{Ha}\end{array}$
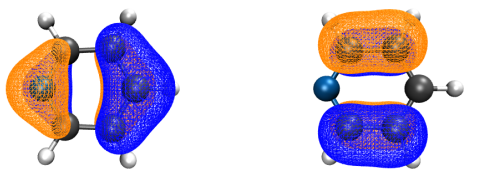

20
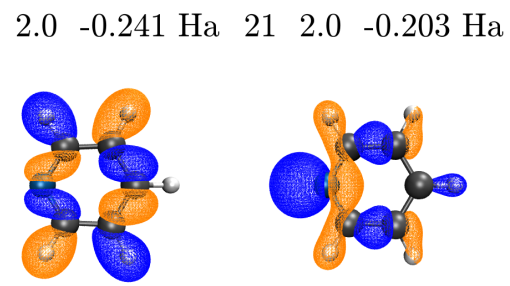

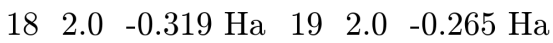

Figure 8. Visualization of the 18th to 26th KS-MO (out of 94) of pyridine in the $6-31 \mathrm{G}^{*}$ basis. The orbital coefficients, energies and occupation numbers were obtained using PYSCF with a RKS-DFT calculation and the LDA/VWN XCF. All orbitals are rendered using VMD 69] at an isovalue of 0.05 . The three numbers below each orbital correspond to the HFMOs index (as indicated in Fig. 7), the occupation number, and the orbital energy, respectively. corrections $\tilde{\varepsilon}_{A S}^{e m b}$ (cf. Eq. (17)) obtained with the DFT and $\mathrm{HF}$ embedding schemes as well as the classical reference, CASSCF.

It should be noted that the DFT embedding scheme requires multiple VQE calculations due to its iterative nature. While this poses a computational burden, the potential benefits in terms of accuracy that can be obtained for more complex systems will be important and will justify the additional computational costs.

\section{CONCLUSIONS}

In this work, we introduced an embedding scheme that enables the partitioning of electronic structure calculations into an Active Space (AS) subsystem treated with a high level quantum algorithm and an environment described at the HF or DFT level of theory. In this way, we can restrict the quantum calculations to a critical subset of molecular orbitals that can fit on state-of-the-art quantum computers, while the remaining electrons provide the embedding potential computed using a classical algorithm. Since in most chemical processes, the quality of the electronic structure predictions depends on a small set of frontier orbitals, this scheme will allow the solution of interesting quantum chemistry problems where the AS can be described with a quantum algorithm presenting a favorable scaling in the number of active electrons. We show the performance of the embedding scheme in the case of a few test molecular systems, namely $\mathrm{H}_{2} \mathrm{O}, \mathrm{N}_{2}$, $\mathrm{CH}_{2}, \mathrm{O}_{2}$ and pyridine, highlighting the benefits of the recursive update of the embedding potential for an improved convergence of the computed ground state energies. It is important to mention that the use of the iterative Range-Separated DFT embedding requires the tuning of an extra parameter, which cannot be set a priori. Further investigation is needed to automatize this technique for general use in larger molecular systems.

Of particular relevance are the results obtained for pyridine; in this case we showed that the iterative quantum computing embedding scheme is able to outperform classical active space methods such as CASSCF using a 


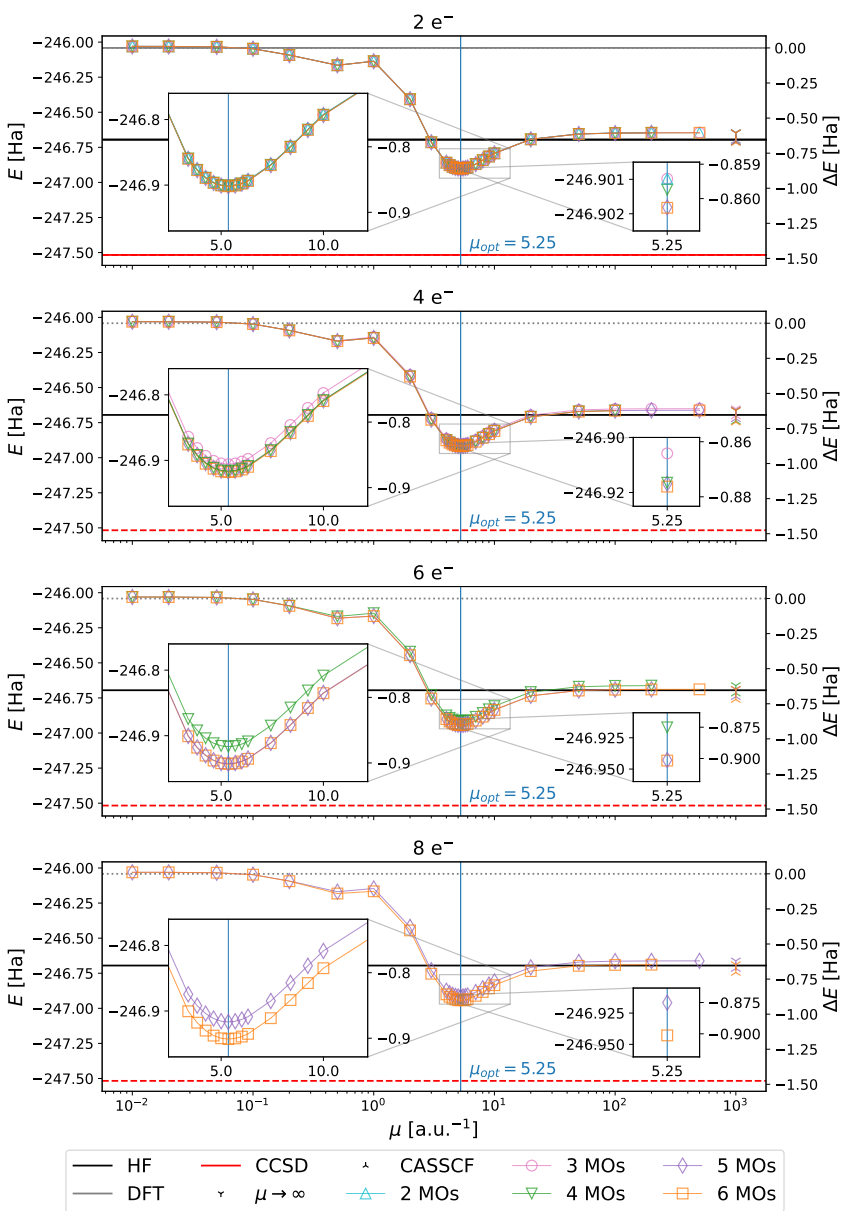

Figure 9. Electronic structure energies (in Hartree) of a pyridine $\left(\mathrm{C}_{5} \mathrm{H}_{5} \mathrm{~N}\right)$ molecule obtained using the DFT embedding scheme for a different number of active electrons. Each panel groups results obtained for different numbers of active electrons (from the top: 2, 4, 6, 8) while the number of MOs in the AS is color-coded (see legend). The classical references were computed using PYSCF and the $6-31 \mathrm{G}^{*}$ basis. The initial density was obtained using RKS-DFT with the LDA/VWN $\mathrm{XCF}$ (grey, dotted). For completeness, we also provide the $\mathrm{HF}$ and CCSD references in solid black and dashed red, respectively. The range separation parameter, $\mu$, is varied along the $x$ axis. Reference values for $\mu \rightarrow \infty$ and for the CASSCF results are included at $\mu=10^{3}$ with different marker symbols and color-coded according to the respective ASs.

reasonable small number of quantum resources (i.e., the DFT embedding scheme with the q-UCCSD expansion of the AS recovers $13 \%$ more energy than the CASSCF approach with the same AS).

Improvements on the proposed embedding scheme can be obtained through the combination of the iterative update of the embedding potential together with the simultaneous optimization of the active orbitals as done, for instance, in the multiconfigurational self-consistent field (MCSCF) approach.

We believe that the proposed HF and DFT embedding schemes will provide a fundamental framework for the scaling up of quantum electronic structure calculations to large molecular systems with an arbitrary number of electrons (i.e., as many as a HF or DFT calculations can deal with). The possibility of partitioning the solution of the electronic structure problem into an active component (defined by the AS) treated by means of a quantum computing algorithm and an inert environment component solved at the HF or DFT level of theory will open up new avenues for the use of quantum computers in the solution of important problems in physics, chemistry, biology and medicine.

\section{ACKNOWLEDGEMENTS}

The authors thank Valery Weber and Jürg Hutter for useful discussions as well as Manfred Sigrist who advised M. R. during a significant part of this work.

I.T. and P.J.O acknowledge financial support from the Swiss National Science Foundation (SNF) through grant No. 200021-179312.

\section{AIP PUBLISHING DATA SHARING POLICY}

The data that support the findings of this study are available from the corresponding author upon reasonable request.

\section{Appendix A: Hartree-Fock Embedding}

In this section we provide more detailed derivations of the $\mathrm{HF}$ embedding. First, we derive the restricted spin case and generalize the equations for unrestricted spins in the second part of this section.

\section{Restricted Spins}

We introduce the splitting of the one-electron density matrix, $D=D^{A}+D^{I}$, into Eq. (1) one term at a time. The simplest case is the one of the one-electron contribution which becomes

$$
\begin{aligned}
\sum_{p q} h_{p q} D_{p q} & =\sum_{v q} h_{v q} D_{v q}^{A}+\sum_{j q} h_{j q} D_{j q}^{I} \\
& =\sum_{u v} h_{u v} D_{u v}^{A}+2 \sum_{j} h_{j j}
\end{aligned}
$$

where we use the fact that a density matrix element vanishes when any of its indices correspond to a virtual orbital. We can proceed analogously with the two-electron 
terms as in

$$
\begin{aligned}
\frac{1}{2} \sum_{p q r s} g_{p q r s} d_{p q r s} & =\frac{1}{2} \sum_{p q j s} g_{p q j s} d_{p q j s}+\frac{1}{2} \sum_{j q u s} g_{j q u s} d_{j q u s} \\
& +\frac{1}{2} \sum_{v q u s} g_{v q u s} d_{v q u s} .
\end{aligned}
$$

We can now express the two-electron density matrices in terms of one-electron ones,

$$
\begin{aligned}
d_{p q j s} & =D_{p q} D_{j s}-\delta_{q j} D_{p s} \\
& =2 \delta_{j s} D_{p q}-\delta_{q j} \delta_{s q} D_{p q} \\
& =\left(2 \delta_{j s}-\delta_{q j} \delta_{s q}\right) D_{p q}
\end{aligned}
$$

and

$$
\begin{aligned}
d_{j q u s} & =d_{u s j q}=D_{u s} D_{j q}-\delta_{s j} D_{u q} \\
& =2 \delta_{j q} D_{u s}-\delta_{s j} D_{u q} \\
& =2 \delta_{q j} \delta_{s q} D_{u q}-\delta_{s j} D_{u q} \\
& =\left(2 \delta_{q j} \delta_{s q}-\delta_{s j}\right) D_{u q},
\end{aligned}
$$

where we omit the superscripts $I$ and $A$ for brevity. These expressions can then be inserted into Eq. A2 to obtain

$$
\begin{aligned}
\frac{1}{2} \sum_{p q r s} g_{p q r s} d_{p q r s} & =\sum_{p q j} g_{p q j j} D_{p q}-\frac{1}{2} \sum_{p q j} g_{p j j q} D_{p q} \\
& +\sum_{j q u} g_{j j u q} D_{u q}^{A}-\frac{1}{2} \sum_{j q u} g_{j q u j} D_{u q}^{A} \\
& +\frac{1}{2} \sum_{u v x y} g_{u v x y} d_{u v x y}^{A} .
\end{aligned}
$$

Upon inspection, it becomes clear that the first two sums only yield non-zero contributions when $p=q=k$ or when $p=u$ and $q=v$. In the latter case, this causes the sums to coincide with the third and forth term. These observations allow us to simplify Eq. A4 to become

$$
\begin{aligned}
\frac{1}{2} \sum_{p q r s} g_{p q r s} d_{p q r s} & =2 \sum_{k j}\left(g_{k k j j}-\frac{1}{2} g_{k j j k}\right) \\
& +2 \sum_{j v u}\left(g_{j j u v}-\frac{1}{2} g_{j v u j}\right) D_{u v}^{A} \\
& +\frac{1}{2} \sum_{u v x y} g_{u v x y} d_{u v x y}^{A} .
\end{aligned}
$$

Finally, we can substitute Eq. A1 and Eq. A5 into Eq. (1) yielding

$$
\begin{aligned}
E & =\sum_{j}\left[2 h_{j j}+\sum_{k}\left(2 g_{k k j j}-g_{k j j k}\right)\right] \\
& +\sum_{u v}\left[h_{u v}+\sum_{j}\left(2 g_{j j u v}-g_{j v u j}\right)\right] D_{u v}^{A} \\
& +\frac{1}{2} \sum_{u v x y} g_{u v x y} d_{u v x y}^{A} .
\end{aligned}
$$

This equation simplifies to Eq. (2) with the use of the inactive Fock operator, Eq. (3), and the inactive energy, Eq. (4).

\section{Unrestricted Spins}

For unrestricted spins we have to remove the implicit summation over the spin state, $\sigma$, and calculate with the one- and two-electron density matrices for each spin separately,

$$
D_{p q}^{\sigma}=\left\langle\Psi\left|\hat{E}_{p q}^{\sigma}\right| \Psi\right\rangle \quad \forall \sigma \in\{\alpha, \beta\}
$$

$$
\begin{array}{cr}
d_{p q r s}^{\sigma \tau}=\left\langle\Psi\left|\hat{e}_{p q r s}^{\sigma \tau}\right| \Psi\right\rangle & \forall \sigma, \tau \in\{\alpha, \beta\} \\
\hat{E}_{p q}^{\sigma}=\hat{a}_{p \sigma}^{\dagger} \hat{a}_{q \sigma} & \forall \sigma \in\{\alpha, \beta\} \\
& (\mathrm{A} 7 \mathrm{~b} 7 \mathrm{c}) \\
\hat{e}_{p q r s}^{\sigma \tau} & =\left\{\begin{array}{llr}
\hat{E}_{p q}^{\sigma} \hat{E}_{r s}^{\sigma}-\delta_{q r} \hat{E}_{p s}^{\sigma}, & \sigma=\tau \\
\hat{E}_{p q}^{\sigma} \hat{E}_{r s}^{\tau} & , \sigma \neq \tau
\end{array}\right.
\end{array}
$$

Thus, in contrast to the closed shell description with restricted spins in the previous section, we now have to keep track of the spin state which each index iterates over. This is indicated by the additional superscripts, $\sigma$ and $\tau$, and summation labels, $n_{\sigma}$ and $n_{\tau}$, respectively. For brevity we refrain from explicitly denoting that $\sigma, \tau \in\{\alpha, \beta\}$ for the remainder of this derivation.

Analogous to Eq. A1 the one-electron contribution can be written per spin state as

$$
\sum_{p q}^{n_{\sigma}} h_{p q} D_{p q}^{\sigma}=\sum_{u v}^{n_{\sigma}} h_{u v} D_{u v}^{A, \sigma}+\sum_{j}^{n_{\sigma}} h_{j j} .
$$

For the two-electron contributions we have to differentiate between two cases. In the first case, the spins of both electrons are aligned, $\sigma=\tau$, and the resulting equation can be derived in full analogy to Eq. A5,

$$
\begin{aligned}
\frac{1}{2} \sum_{p q}^{n_{\sigma}} \sum_{r s}^{n_{\sigma}} g_{p q r s} d_{p q r s}^{\sigma \sigma} & =\frac{1}{2} \sum_{k}^{n_{\sigma}} \sum_{j}^{n_{\sigma}}\left(g_{k k j j}-g_{k j j k}\right) \\
& +\sum_{j}^{n_{\sigma}} \sum_{u v}^{n_{\sigma}}\left(g_{j j u v}-g_{j v u j}\right) D_{u v}^{A, \sigma} \\
& +\frac{1}{2} \sum_{u v}^{n_{\sigma}} \sum_{x y}^{n_{\sigma}} g_{u v x y} d_{u v x y}^{A, \sigma \sigma} .
\end{aligned}
$$

The second case of opposite spins, $\sigma \neq \tau$, behaves slightly different due to the differing two-electron excitation operator, Eq. A7d. Thus, the expression of the twoelectron density matrices in terms of one-electron ones analogous to Eq. A3 becomes

$$
d_{p q j s}^{\sigma \tau}=D_{p q}^{\sigma} D_{j s}^{\tau}=\delta_{j s} D_{p q}^{\sigma}
$$


and

$$
d_{j q u s}^{\sigma \tau}=D_{j q}^{\sigma} D_{u s}^{\tau}=\delta_{j q} D_{u s}^{\tau},
$$

which leads to the first expression of the two-electron contribution of opposite spins

$$
\begin{aligned}
\frac{1}{2} \sum_{p q}^{n_{\sigma}} \sum_{r s}^{n_{\tau}} g_{p q r s} d_{p q r s}^{\sigma \tau} & =\frac{1}{2} \sum_{p q}^{n_{\sigma}} \sum_{j}^{n_{\tau}} g_{p q j j} D_{p q}^{\sigma} \\
& +\frac{1}{2} \sum_{j}^{n_{\sigma}} \sum_{u s}^{n_{\tau}} g_{j j u s} D_{u s}^{A, \tau} \\
& +\frac{1}{2} \sum_{v q}^{n_{\sigma}} \sum_{u s}^{n_{\tau}} g_{v q u s} d_{v q u s}^{A, \sigma \tau} .
\end{aligned}
$$

Using the same arguments as before we can once again simplify this equation to become

$$
\begin{aligned}
\frac{1}{2} \sum_{p q}^{n_{\sigma}} \sum_{r s}^{n_{\tau}} g_{p q r s} d_{p q r s}^{\sigma \tau}= & \\
= & \frac{1}{2} \sum_{k}^{n_{\sigma}} \sum_{j}^{n_{\tau}} g_{k k j j}+\frac{1}{2} \sum_{u v}^{n_{\sigma}} \sum_{j}^{n_{\tau}} g_{u v j j} D_{u v}^{A, \sigma} \\
& +\frac{1}{2} \sum_{j}^{n_{\sigma}} \sum_{u v}^{n_{\tau}} g_{j j u v} D_{u v}^{A, \tau}+\frac{1}{2} \sum_{u v}^{n_{\sigma}} \sum_{x y}^{n_{\tau}} g_{u v x y} d_{u v x y}^{A, \sigma \tau} .
\end{aligned}
$$

Finally, we can obtain the two-electron contributions for unrestricted spins by combining Eq. A9) and Eq. A12 into

$$
\begin{aligned}
\frac{1}{2} \sum_{p q r s} g_{p q r s} d_{p q r s}= \\
=\frac{1}{2} \sum_{j}^{n_{\alpha}}\left[\sum_{k}^{n_{\alpha}}\left(g_{k k j j}-g_{k j j k}\right)+\sum_{k}^{n_{\beta}} g_{k k j j}\right] \\
+\frac{1}{2} \sum_{j}^{n_{\beta}}\left[\sum_{k}^{n_{\beta}}\left(g_{k k j j}-g_{k j j k}\right)+\sum_{k}^{n_{\alpha}} g_{k k j j}\right] \\
+\sum_{u v}^{n_{\alpha}}\left[\sum_{j}^{n_{\alpha}}\left(g_{u v j j}-g_{u j j v}\right)+\sum_{j}^{n_{\beta}} g_{u v j j}\right] D_{u v}^{A, \alpha} \\
+\sum_{u v}^{n_{\beta}}\left[\sum_{j}^{n_{\beta}}\left(g_{u v j j}-g_{u j j v}\right)+\sum_{j}^{n_{\alpha}} g_{u v j j}\right] D_{u v}^{A, \beta} \\
+\frac{1}{2} \sum_{u v x y} g_{u v x y} d_{u v x y}^{A} .
\end{aligned}
$$

In full analogy to the restricted spin case this allows us to define the inactive Fock operator and energy as

$$
F_{p q}^{I, \sigma}=h_{p q}+\sum_{i}^{n_{\sigma}}\left(g_{i i p q}-g_{i q p i}\right)+\sum_{i}^{n_{\tau}} g_{i i p q}
$$

and

$$
\begin{aligned}
E^{I} & =\frac{1}{2} \sum_{\sigma=\alpha}^{\beta} \sum_{j} h_{j j}+F_{j j}^{I, \sigma} \\
& =\frac{1}{2} \sum_{\sigma=\alpha}^{\beta} \sum_{i j}\left(h_{i j}+F_{i j}^{I, \sigma}\right) D_{i j}^{I, \sigma} .
\end{aligned}
$$

\section{Appendix B: Density Functional Theory Embedding}

In this section, we provide more detailed steps deriving the embedding equations of the iterative DFT embedding scheme. In doing so, we follow the work of Hedegård et. al. 54 rather closely. We focus on the steps necessary to arrive at the final form of the total electronic energy, Eq. (15), after the introduction of the linear model, Eq. 13.

We start by noting that $\Delta D_{p q}^{(i)}=\Delta D_{u v}^{A,(i)}$ since the inactive part of the density matrix, $D_{i j}^{I}$, is constant by definition. Thus, we can express the total electronic energy after exploiting inherent properties of the one-electron density matrices as

$$
\begin{aligned}
E & =E^{I, \mathrm{LR}}+E^{A,(i+1), \mathrm{LR}}+E_{\mathrm{coul}+\mathrm{xc}}^{\mathrm{SR}}\left[\rho^{(i)}\right] \\
& +\sum_{u v}\left(j_{u v}^{(i), \mathrm{SR}}+\nu_{\mathrm{xc}, u v}^{\mathrm{SR}}\left[\rho^{(i)}\right]\right) \Delta D_{u v}^{A,(i)} .
\end{aligned}
$$

To ease the implementation of Eq. (B1) we can rewrite the equation and group its terms into active and inactive ones. To do so, we start by rewriting the Coulomb part of the third term by expanding $D^{(i)}=D^{I}+D^{A,(i)}$ twice,

$$
\begin{aligned}
J^{\mathrm{SR}}\left[\rho^{(i)}\right] & =\frac{1}{2} \sum_{p q r s} D_{p q}^{(i)} g_{p q r s}^{\mathrm{SR}} D_{r s}^{(i)} \\
& =\frac{1}{2} \sum_{p q} D_{p q}^{(i)}\left(\sum_{i j} g_{p q i j}^{\mathrm{SR}} D_{i j}^{I}+\sum_{u v} g_{p q u v}^{\mathrm{SR}} D_{u v}^{A,(i)}\right) \\
& =\frac{1}{2}\left(\sum_{k l i j} D_{k l}^{I} g_{k l i j}^{\mathrm{SR}} D_{i j}^{I}+\sum_{x y i j} D_{x y}^{A,(i)} g_{x y i j}^{\mathrm{SR}} D_{i j}^{I}\right. \\
& \left.+\sum_{k l u v} D_{k l}^{I} g_{k l u v}^{\mathrm{SR}} D_{u v}^{A,(i)}+\sum_{x y u v} D_{x y}^{A,(i)} g_{x y u v}^{\mathrm{SR}} D_{u v}^{A,(i)}\right) .
\end{aligned}
$$

We can proceed analogously with the Coulomb part of the fourth term,

$$
\begin{gathered}
\sum_{u v} j_{u v}^{(i), \mathrm{SR}} \Delta D_{u v}^{A,(i)}=\sum_{p q u v} D_{p q}^{(i)} g_{p q u v}^{\mathrm{SR}}\left(D_{u v}^{A,(i+1)}-D_{u v}^{A,(i)}\right) \\
=\sum_{i j u v} D_{i j}^{I} g_{i j u v}^{\mathrm{SR}} D_{u v}^{A,(i+1)}-D_{i j}^{I} g_{i j u v}^{\mathrm{SR}} D_{u v}^{A,(i)} \\
\quad+\sum_{x y u v} D_{x y}^{A,(i)} g_{x y u v}^{\mathrm{SR}} D_{u v}^{A,(i+1)}-D_{x y}^{A,(i)} g_{x y u v}^{\mathrm{SR}} D_{u v}^{A,(i)} .
\end{gathered}
$$


By gathering and canceling matching terms of Eq. B2 and Eq. (B3) through the use of the symmetry, $g_{p q r s}=$ $g_{r s p q}$, we arrive at the final expression of the SR Coulomb contributions

$$
\begin{aligned}
J^{\mathrm{SR}} & {\left[\rho^{(i)}\right]+\sum_{u v} j_{u v}^{(i), \mathrm{SR}} \Delta D_{u v}^{A,(i)} } \\
& =\frac{1}{2} \sum_{i j} j_{i j}^{I, \mathrm{SR}} D_{i j}^{I}-\frac{1}{2} \sum_{u v} j_{u v}^{A,(i), \mathrm{SR}} D_{u v}^{A,(i)} \\
& +\sum_{u v} j_{u v}^{I, \mathrm{SR}} D_{u v}^{A,(i+1)}+\sum_{u v} j_{u v}^{A,(i), \mathrm{SR}} D_{u v}^{A,(i+1)} .
\end{aligned}
$$

Inserting Eq. (B4) into Eq. (B1) finally leads to

$$
\begin{aligned}
E & =E^{I, \mathrm{LR}}+E_{\mathrm{xc}}^{\mathrm{SR}}\left[\rho^{(i)}\right]+\frac{1}{2} \sum_{i j} j_{i j}^{I, \mathrm{SR}} D_{i j}^{I} \\
& -\frac{1}{2} \sum_{u v} j_{u v}^{A,(i), \mathrm{SR}} D_{u v}^{A,(i)}-\sum_{u v} \nu_{\mathrm{xc}, u v}^{\mathrm{SR}}\left[\rho^{(i)}\right] D_{u v}^{A,(i)} \\
& +E^{A, \mathrm{LR}}+\sum_{u v} j_{u v}^{I, \mathrm{SR}} D_{u v}^{A,(i+1)}+\sum_{u v} j_{u v}^{A,(i), \mathrm{SR}} D_{u v}^{A,(i+1)} \\
& +\sum_{u v} \nu_{\mathrm{xc}, u v}^{\mathrm{SR}}\left[\rho^{(i)}\right] D_{u v}^{A,(i+1)}
\end{aligned}
$$

where we have re-ordered the terms such that the upper two lines contain all the inactive terms and the lower lines all the active ones. After insertion of the expressions for the LR energy contributions, $E^{I}$ and $E^{A}$, according to Eq. (4) and Eq. (2), respectively, we arrive at the final expression of the total electronic energy, Eq. (15).

The extension of these equations to unrestricted spins is similarly straight forward as in the case of the $\mathrm{HF}$ embedding (cf. Appendix A 2).
[1] J. D. Whitfield, J. Biamonte, and A. Aspuru-Guzik, Mol. Phys. 109, 735 (2011).

[2] T. Helgaker, S. Coriani, P. Jørgensen, K. Kristensen, J. Olsen, and K. Ruud, Chem. Rev. 112, 543 (2012).

[3] K. D. Vogiatzis, M. V. Polynski, J. K. Kirkland, J. Townsend, A. Hashemi, C. Liu, and E. A. Pidko, Chem. Rev. 119, 2453 (2018).

[4] M. Reiher, N. Wiebe, K. M. Svore, D. Wecker, and M. Troyer, Proc. Natl. Acad. Sci. 114, 7555 (2017).

[5] M. Boero, M. Parrinello, and K. Terakura, J. Am. Chem. Soc. 120, 2746 (1998).

[6] A. Aspuru-Guzik, R. Lindh, and M. Reiher, ACS Cent. Sci. 4, 144 (2018).

[7] Y. CaO, J. Romero, and A. Aspuru-Guzik, IBM J. Res. Dev. 62, 6:1 (2018).

[8] J.-M. Reiner, F. Wilhelm-Mauch, G. Schön, and M. Marthaler, Quantum Sci. Technol. 4, 035005 (2019).

[9] I. O. Sokolov, P. K. Barkoutsos, P. J. Ollitrault, D. Greenberg, J. Rice, M. Pistoia, and I. TaverNElli, J. Chem. Phys. 152, 124107 (2020).

[10] C. Kokail, C. Maier, R. van Bijnen, T. Brydges, M. K. Joshi, P. Jurcevic, C. A. Muschik, P. Silvi, R. Blatt, C. F. Roos, and P. Zoller, Nature 569, 355 (2019).

[11] S. V. Mathis, G. Mazzola, and I. Tavernelli, Toward scalable simulations of Lattice Gauge Theories on quantum computers, e-print: arXiv:2005.10271 v1, 2020.

[12] R. Babbush, N. Wiebe, J. McClean, J. McClain, H. Neven, and G. K.-L. Chan, Phys. Rev. X 8, 011044 (2018).

[13] IBM, IBM Quantum Experience, https://quantum- computing.ibm.com/ accessed: 2020-03-16.

[14] D. Wecker, M. B. Hastings, and M. Troyer, Phys. Rev. A 92, 042303 (2015).

[15] A. Peruzzo, J. McClean, P. Shadbolt, M.-H. Yung, X.-Q. Zhou, P. J. Love, A. Aspuru-Guzik, and J. L. O'Brien, Nat. Commun. 5, 4213 (2014).

[16] J. Č́́žEk, J. Chem. Phys. 45, 4256 (1966).

[17] H. G. KüMMEL, A biography of the coupled cluster method, in Recent progress in many-body theories Proceedings of the 11th international conference., edited by R. F. Bishop, T. Brandes, K. A. Gernoth, N. R. Walet, and Y. Xian, pp. 334-348, World Scientific Publishing, Singapore, 2002.

[18] R. J. Bartlett and M. Musial, Rev. Mod. Phys. 79, 291 (2007).

[19] P. O'Malley, R. Babbush, I. Kivlichan, J. Romero, J. McClean, R. Barends, J. Kelly, P. Roushan, A. Tranter, N. Ding, B. Campbell, Y. Chen, Z. Chen, B. Chinro, A. Dunsworth, A. Fowler, E. Jeffrey, E. Lucero, A. Megrant, J. Mutus, M. Neeley, C. Neill, C. Quintana, D. Sank, A. Vainsencher, J. Wenner, T. White, P. Coveney, P. Love, H. Neven, A. Aspuru-GuziK, and J. MartiNIS, Phys. Rev. X 6, 031007 (2016).

[20] J. Romero, R. Babbush, J. R. McClean, C. Hempel, P. Love, and A. Aspuru-GuzIK, Quantum Sci. Technol. 4, 014008 (2018).

[21] P. K. Barkoutsos, J. F. Gonthier, I. Sokolov, N. Moll, G. Salis, A. Fuhrer, M. Ganzhorn, D. J. Egger, M. Troyer, A. Mezzacapo, S. Filipp, and I. Tavernelli, Phys. Rev. A 98, 022322 (2018).

[22] N. Moll, P. Barkoutsos, L. S. Bishop, J. M. Chow, A. Cross, D. J. Egger, S. Filipp, A. Fuhrer, J. M. 
Gambetta, M. Ganzhorn, A. Kandala, A. MezzaCAPo, P. Müller, W. Riess, G. Salis, J. Smolin, I. TAVERnelli, and K. Temme, Quantum Sci. Technol. 3, 030503 (2018).

[23] M. Kühn, S. Zanker, P. Deglmann, M. Marthaler, and H. Weiss, J. Chem. Theory Comput. 15, 4764 (2019).

[24] N. P. Bauman, E. J. Bylaska, S. Krishnamoorthy, G. H. Low, N. Wiebe, C. E. Granade, M. Roetteler, M. Troyer, and K. Kowalski, J. Chem. Phys. 151, 014107 (2019).

[25] F. A. Evangelista, G. K.-L. Chan, and G. E. ScuseRIA, J. Chem. Phys. 151, 244112 (2019).

[26] W. Mizukami, K. Mitarai, Y. O. Nakagawa, T. YaMAMOTO, T. YAN, and Y. YA OHNISHI, Orbital optimized unitary coupled cluster theory for quantum computer, eprint arXiv:1910.11526v3, 2019.

[27] A. Kandala, A. Mezzacapo, K. Temme, M. Takita, M. Brink, J. M. Chow, and J. M. Gambetta, Nature 549, 242 (2017).

[28] C. Hempel, C. Maier, J. Romero, J. McClean, T. Monz, H. Shen, P. Jurcevic, B. P. Lanyon, P. Love, R. Babbush, A. Aspuru-Guzik, R. Blatt, and C. F. Roos, Phys. Rev. X 8, 031022 (2018).

[29] R. Sagastizabal, X. Bonet-Monroig, M. Singh, M. A. Rol, C. C. Bultink, X. Fu, C. H. Price, V. P. Ostroukh, N. Muthusubramanian, A. Bruno, M. Beekman, N. Haider, T. E. O'Brien, and L. DiCarlo, Phys. Rev. A 100, 010302 (2019).

[30] F. Arute, K. Arya, R. Babbush, D. Bacon, J. C. Bardin, R. Barends, S. Boixo, M. Broughton, B. B. Buckley, D. A. Buell, B. Burkett, N. Bushnell, Y. Chen, Z. Chen, B. Chiaro, R. Collins, W. Courtney, S. Demura, A. Dunsworth, D. EpPens, E. Farhi, A. Fowler, B. Foxen, C. Gidney, M. Giustina, R. Graff, S. Habegger, M. P. HarRigan, A. Ho, S. Hong, T. Huang, W. J. Huggins, L. Ioffe, S. V. IsAkov, E. JefFrey, Z. JiAng, C. Jones, D. Kafri, K. Kechedzhi, J. Kelly, S. Kim, P. V. Klimov, A. Korotkov, F. Kostritsa, D. Landhuis, P. Laptev, M. Lindmark, E. Lucero, O. Martin, J. M. Martinis, J. R. McClean, M. McEwen, A. Megrant, X. Mi, M. Mohseni, W. Mruczkiewicz, J. Mutus, O. NaAman, M. Neeley, C. Neill, H. Neven, M. Y. Niu, T. E. O'Brien, E. Ostby, A. Petukhov, H. Putterman, C. Quintana, P. Roushan, N. C. Rubin, D. Sank, K. J. Satzinger, V. Smelyanskiy, D. Strain, K. J. Sung, M. SzaLAY, T. Y. TAKeshita, A. Vainsencher, T. White, N. Wiebe, Z. J. YaO, P. Yeh, and A. Zalcman, Hartree-Fock on a superconducting qubit quantum computer, e-print arXiv:2004.04174 v3, 2020.

[31] P. J. Ollitrault, A. Kandala, C.-F. Chen, P. K. Barkoutsos, A. Mezzacapo, M. Pistoia, S. ShelDon, S. Woerner, J. Gambetta, and I. TAVernelli, Quantum equation of motion for computing molecular excitation energies on a noisy quantum processor, e-print: arXiv:1910.12890, 2019.

[32] O. Higgott, D. Wang, and S. Brierley, Quantum 3, 156 (2019).

[33] R. M. Parrish, E. G. Hohenstein, P. L. McMahon, and T. J. Martinez, Phys. Rev. Lett. 122, 230401 (2019).

[34] K. M. Nakanishi, K. Mitarai, and K. Fujil, Phys.
Rev. Res. 1, 033062 (2019).

[35] I. G. Ryabinkin, S. N. Genin, and A. F. Izmaylov, J. Chem. 15, 249 (2018).

[36] J. R. McClean, M. E. Kimchi-Schwartz, J. Carter, and W. A. DE Jong, Phys. Rev. A 95, 042308 (2017).

[37] N. H. Stair, R. Huang, and F. A. Evangelista, J. Chem. 16, 2236 (2020).

[38] R. Santagati, J. Wang, A. A. Gentile, S. Paesani, N. Wiebe, J. R. McClean, S. Morley-Short, P. J. Shadbolt, D. Bonneau, J. W. Silverstone, et al., Sci. Adv. 4, eaap9646 (2018).

[39] T. Jones, S. Endo, S. McArdle, X. Yuan, and S. C. Benjamin, Phys. Rev. A 99, 062304 (2019).

[40] J. Tilly, G. Jones, H. Chen, L. Wossnig, and E. GRANT, Computation of molecular excited states on IBMQ using a Discriminative Variational Quantum Eigensolver, 2020, e-print: arXiv:2001.04941

[41] B. O. Roos, P. R. Taylor, and P. E. Sigbahn, Chem. Phys. 48, 157 (1980).

[42] T. Helgaker, P. Jørgensen, and J. Olsen, Molecular Electronic-Structure Theory, John Wiley \& Sons, Ltd, 2000.

[43] B. Bauer, D. Wecker, A. J. Millis, M. B. Hastings, and M. Troyer, Phys. Rev. X 6, 031045 (2016).

[44] N. C. Rubin, A Hybrid Classical/Quantum Approach for Large-Scale Studies of Quantum Systems with Density Matrix Embedding Theory, e-print: arXiv:1610.06910v2, 2016.

[45] A. Georges and G. Kotliar, Phys. Rev. B 45, 6479 (1992).

[46] G. Knizia and G. K.-L. Chan, Phys. Rev. Lett. 109, $186404(2012)$

[47] X. Wu, M. Lindsey, T. Zhou, Y. Tong, and L. LIN, Enhancing robustness and efficiency of density matrix embedding theory via semidefinite programming and local correlation potential fitting, e-print: arXiv:2003.00873v1, 2020.

[48] H. Ma, M. Govoni, and G. Galli, NPJ Comput. Mater. 6 (2020).

[49] A. SAvin and H.-J. FlaD, Int. J. Quantum Chem. 56, 327 (1995).

[50] T. Takeshita, N. C. Rubin, Z. Jiang, E. Lee, R. BabBush, and J. R. MCClean, Phys. Rev. X 10, 011004 (2020).

[51] M. Urbanek, D. Camps, R. V. Beeumen, and W. A. DE Jong, Chemistry on quantum computers with virtual quantum subspace expansion, e-print: arXiv:2002.12902 $1,2020$.

[52] G. Aleksandrowicz, T. Alexander, P. Barkoutsos, L. Bello, Y. Ben-Haim, D. Bucher, F. J. CabreraHernandez, J. Carballo-Franquis, A. Chen, C.F. Chen, J. M. Chow, A. D. Corcoles-Gonzales, A. J. Cross, A. Cross, J. Cruz-Benito, C. CulVer, S. D. L. P. Gonzalez, E. D. L. Torre, D. Ding, E. Dumitrescu, I. Duran, P. EenDebak, M. Everitt, I. F. Sertage, A. Frisch, A. Fuhrer, J. Gacon, J. Gambetta, B. G. Gago, J. Gomez-Mosquera, D. Greenberg, I. Hamamura, V. Havlicek, J. Hellmers, L. Herok, H. Horit, S. Hu, T. Imamichi, T. Itoko, A. JavadiAbhari, N. Kanazawa, A. Karazeev, K. Krsulich, P. Liu, Y. Luh, Y. Maeng, M. Marques, F. J. Martin-Fernandez, D. T. McClure, D. MCKay, S. Meesala, A. Mezzacapo, N. Moll, D. M. Ro- 
Driguez, G. Nannicini, P. Nation, P. J. Ollitrault, L. J. O'Riordan, H. Paik, J. Perez, A. Phan, M. Pistoia, V. Prutyanov, M. Reuter, J. Rice, A. R. Davila, M. Rossmannek, R. H. P. Rudy, M. Ryu, N. Sathaye, C. Schnabel, E. Schoute, K. Setia, Y. Shi, A. Silva, Y. Siraichi, S. Sivarajah, J. A. Smolin, M. Soeken, I. Sokolov, H. Takahashi, I. Tavernelli, C. TAYlor, P. TAylour, K. Trabing, M. Treinish, W. Turner, D. Vogt-Lee, C. Vuillot, J. A. Wildstrom, J. Wilson, E. Winston, C. Wood, S. Wood, S. Wörner, I. Y. Akhalwaya, and C. ZoUFAL, Qiskit: An Open-source Framework for Quantum Computing, 2019, DOI:10.5281/zenodo.2562111.

[53] S. B. Bravyi and A. Y. Kitaev, Ann. Phys. (N Y) 298, 210 (2002).

[54] E. D. Hedegånd, S. Knecht, J. S. Kielberg, H. J. A. Jensen, and M. Reiher, J. Chem. Phys. 142, 224108 (2015).

[55] QISkIT, Qiskit Aqua, https://github.com/Qiskit/qiskitaqua/ accessed: 2020-08-18.

[56] Q. Sun, J. Yang, and G. K.-L. Chan, Chem. Phys. Lett. 683, 291 (2017).

[57] The ExactEigensolver method was renamed to NumPyEigensolver in QISKIT Aqua's 0.7 release.

[58] S. Bravyi, J. M. Gambetta, A. Mezzacapo, and K. Temme, Tapering off qubits to simulate fermionic Hamiltonians, e-print: arXiv:1701.08213 $1,2017$.

[59] J. L. Morales and J. Nocedal, ACM Trans. Math. Softw. 38, 1 (2011).

[60] D. KRAFT, A software package for sequential quadratic programming, DFVLR, Köln, 1988.

[61] J. Toulouse, A. Savin, and H.-J. Flad, Int. J. Quantum Chem. 100, 1047 (2004).

[62] S. Paziani, S. Moroni, P. Gori-Giorgi, and G. B. BAchelet, Phys. Rev. B 73, 155111 (2006).

[63] U. Ekström, L. Visscher, R. Bast, A. J. ThorvaldSEN, and K. Ruud, J. Chem. Theory Comput. 6, 1971 (2010).

[64] J. Heyd, G. E. Scuseria, and M. Ernzerhof, $J$. Chem. Phys. 118, 8207 (2003).

[65] E. Fromager, J. Toulouse, and H. J. A. Jensen, J. Chem. Phys. 126, 074111 (2007).

[66] W. J. Hehre, R. F. Stewart, and J. A. Pople, J. Chem. Phys. 51, 2657 (1969).

[67] P. C. Hariharan and J. A. Pople, Theor. Chim. Acta 28, 213 (1973).

[68] T. H. Dunning, J. Chem. Phys. 90, 1007 (1989).

[69] W. Humphrey, A. Dalke, and K. Schulten, J. Mol. Graph. 14, 33 (1996).

[70] V. Veryazov, P. A. Malmqvist, and B. O. Roos, Int. J. Quantum Chem. 111, 3329 (2011).

[71] C. J. Stein and M. Reiher, J. Chem. Theory Comput. 12, 1760 (2016).

[72] C. J. Stein and M. Reiner, J. Comput. Chem. 40, 2216 (2019).

[73] F. M. Faulstich, M. Máté, A. Laestadius, M. A. Csirik, L. Veis, A. Antalik, J. Brabec, R. SchneiDer, J. Pittner, S. KvaAl, and Ö. Legeza, J. Chem. Theory Comput. 15, 2206 (2019).

[74] E. R. Sayfutyarova, Q. Sun, G. K.-L. Chan, and G. Knizia, J. Chem. Theory Comput. 13, 4063 (2017).

[75] M. Ganzhorn, D. Egger, P. Barkoutsos, P. Ollitrault, G. Salis, N. Moll, M. Roth, A. Fuhrer, P. Mueller, S. Woerner, I. Tavernelli, and S. Fil-
IPP, Phys. Rev. Appl. 11, 044092 (2019).

[76] A. Choquette, A. D. Paolo, P. K. Barkoutsos, D. SÉnéchal, I. TAVERnelli, and A. Blais, Quantumoptimal-control-inspired ansatz for variational quantum algorithms, e-print: arXiv:2008.01098v1, 2020. 